\title{
Relationship between breathing and cardiovascular function at rest: sex-related differences
}

\author{
B. G. Wallin ${ }^{1}$, E. C. Hart ${ }^{2}$, E. A. Wehrwein ${ }^{2}$, N. Charkoudian ${ }^{3}$, and M. J. Joyner ${ }^{2}$ \\ ${ }^{1}$ Institute of Neuroscience and Physiology, The Sahlgrenska Academy at Gothenburg University, \\ Gothenburg, Sweden \\ 2 Department of Anesthesiology, The Mayo Clinic, Rochester, MN, USA \\ ${ }^{3}$ Department of Physiology and Biomedical Engineering, Mayo Clinic, Rochester, MN, USA
}

\begin{abstract}
Aim-To compare relationships at rest between breathing rate, levels of muscle sympathetic nerve activity, total peripheral resistance and cardiac output among young men and women.

Methods-Recordings were made of respiratory movements, sympathetic nerve activity (peroneal microneurography), intra-arterial blood pressure, electrocardiogram, cardiac output (open-circuit acetylene uptake technique) in 19 healthy men (age $27 \pm 2$ years, mean \pm SEM) and 17 healthy women (age $25 \pm 1$ years). Total peripheral resistance and stroke volume were calculated. Four minutes epochs of data were analysed.
\end{abstract}

Results-Breathing rates and sympathetic activity were similar in men and women but compared to men, women had significantly lower blood pressures, cardiac output and stroke volume. In men breathing rate correlated positively with sympathetic activity $(r=0.58, P<0.05)$ but not in women $(r=0.12, P>0.05)$. Furthermore, in men, respiratory rate correlated positively with total peripheral resistance $(r=0.65, P<0.05)$ and inversely with cardiac output $(r=-0.84, P<0.05)$ and heart rate $(r=-0.60, P<0.05)$ but there were no such relationships in women $(P>0.05$ for all).

Conclusions-The positive relationship between breathing and sympathetic activity in men, and the inverse coupling of breathing to cardiac output and heart rate suggest that influences of respiration may be important not only for dynamic but also for 'tonic' cardiovascular function. The lack of relationships among these variables in women shows that there are fundamental differences in basic blood pressure regulation between the sexes.

\section{Keywords}

blood pressure; gender; muscle sympathetic nerve activity; respiration

In a given human subject the strength of resting muscle sympathetic nerve activity (MSNA) is reproducible over several years but among individuals, the strength may vary 7- to 10-fold or more (Sundlöf \& Wallin 1977, Fagius \& Wallin 1993, Charkoudian et al. 2005). Despite the large inter-individual differences in MSNA, there is no relationship between resting levels of MSNA and blood pressure in young healthy individuals of both sexes (Sundlöf \&

Correspondence: Professor B. Gunnar Wallin, Department of Clinical Neurophysiology, Institute of Neuroscience and Physiology, Sahlgrenska University Hospital, S-413 45 Gothenburg, Sweden. gunnar.wallin@ neuro.gu.se.

Conflict of interest

There is no conflict of interest. 
Wallin 1978, Charkoudian et al. 2005, Narkiewicz et al. 2005). In recent studies we investigated this apparent paradox, and found that, in young men, higher levels of MSNA were balanced by lower levels of cardiac output (Charkoudian et al. 2005) and vascular adrenergic responsiveness (Charkoudian et al. 2006). In a follow-up study to evaluate potential sex differences in this neural-haemodynamic balance (Hart et al. 2009) we found marked differences in the relationship between resting MSNA, total peripheral resistance and cardiac output among men and women.

The interaction between respiratory and cardiovascular mechanisms has been investigated mostly with regard to the rhythmic interplay between breathing and autonomic neural and/or cardiovascular function over the respiratory cycle. Studies in anaesthetized animals suggest that respiratory and autonomic neural rhythms originate in the same brainstem area or are driven from a common oscillator in the brainstem (Bachoo \& Polosa 1987, Guyenet et al. 1990). In humans, the close relationships between variations of breathing, sympathetic nerve traffic and cardiovascular measures have been interpreted in terms of a central respiratory gate, rather than baroreflex-related phenomena (Eckberg 2003).

In contrast to the dynamic relationships occurring over the respiratory cycle, little attention has been given to the coupling between inter-individual differences in breathing characteristics, basal levels of sympathetic nerve traffic and cardiovascular function in the resting state. In that context, it is known, however, that at rest the intra-individual reproducibility and the inter-individual differences in resting MSNA, mentioned above, have a respiratory counterpart: the breathing pattern (including breathing rate, tidal volume, inspiratory and expiratory durations) displays marked inter-individual diversity and longterm intra-individual reproducibility (Shea \& Guz 1992, Benchetrit 2000).

These findings may indicate a systematic link between the inherent characteristics of an individual's respiration and his/her baseline sympathetic activity. Recently, this idea was supported by the finding of a positive correlation between spontaneous breathing frequency and strength of MSNA in men (Narkiewicz et al. 2005). Against this background the present study was under-taken to test the hypothesis that the relationships between respiratory frequency, MSNA and cardiovascular variables also differ between men and women.

\section{Material and methods}

\section{Subjects}

The subjects were 19 young men aged 18-35 years and 17 young women aged 19-30 years. Seventeen of the men and 15 of the women were included in Hart et al. (2009) (four men and two women from the material of Hart et al. 2009 were excluded because of inadequate respiratory records). Further characteristics are given in Table 1 . The study protocol was approved by the Institutional Review Board of the Mayo Foundation. The subjects volunteered to participate and gave written informed consent and the studies were completed in accordance with the Declaration of Helsinki.

Participants were asked to not consume anything except small volumes of water within $2 \mathrm{~h}$ of the experiment and were asked to abstain from caffeine or alcohol consumption $24 \mathrm{~h}$ before the study. All women were studied in the early follicular phase of the menstrual cycle or in the low hormone phase of oral contraceptive use (Minson et al. 2000). All subjects were non-smokers, non-diabetic, normally active (neither sedentary nor highly exercise trained) and not currently taking antihypertensive or other medications except for oral contraceptives. Candidates were considered ineligible if they had any acute or chronic disorders associated with alterations in cardiovascular structure or function. 


\section{Measurements}

All studies were performed in a Clinical Research Unit laboratory at the Mayo Clinic maintained at $\sim 22{ }^{\circ} \mathrm{C}$. Upon arrival to the laboratory, subjects rested in the supine position during instrumentation. After local anaesthesia with $2 \%$ lidocaine, a 5-cm, 20-gauge arterial catheter was placed in the brachial artery, using the aseptic technique. This catheter was connected to a pressure transducer placed at the level of the heart and used for measurement of arterial pressure.

A three-lead electrocardiogram (ECG) was used for continuous monitoring of heart rate. A strain gauge belt was wrapped around the upper abdomen to record respiratory movements. Cardiac output was measured using an open-circuit acetylene uptake technique, as described previously (Johnson et al. 2000). This technique has been validated against direct Fick measurements of cardiac outputs over a range of values and has a variability of $\sim 7 \%$ at rest (Johnson et al. 2000). The instrumentation period included a practice cardiac output measurement to familiarize the subject with the procedure.

Multi-unit MSNA was measured from the right peroneal nerve at the fibular head using insulated tungsten microelectrodes (Sundlöf \& Wallin 1977). A muscle sympathetic fascicle was identified when taps on the muscle belly or passive muscle stretch evoked mechanoreceptive impulses, and no afferent neural response was evoked by skin stimuli. The recorded signal was amplified 80000 -fold, band pass filtered $(700-2000 \mathrm{~Hz})$, rectified and integrated (resistance-capacitance integrator circuit time constant $0.1 \mathrm{~s}$ ) by a nerve traffic analyser.

Protocol-After placement of arterial catheters, ECG leads and device for recording respiratory movements, subjects rested supine during instrumentation for microneurography. Once a good electrode site for measurement of MSNA was found, baseline data were recorded for 7-15 min and cardiac output was then determined.

\section{Data analysis}

Data were sampled at $240 \mathrm{~Hz}$ and stored on a personal computer for offline analysis. Mean arterial pressure was calculated as the time integral over the pressure pulse. MSNA, heart rate, mean and diastolic blood pressures were taken as 4 min averages during the $4 \mathrm{~min}$ immediately preceding the first measurement of cardiac output. Breathing rate (breaths $\mathrm{min}^{-1}$ ) was also determined from the same time period. No attention was paid to variation in breathing pattern (regularity and depth) among individuals. Cardiac output is reported as the average of the two measurements for each individual. Total peripheral resistance was calculated as mean blood pressure divided by cardiac output and stroke volume was calculated as cardiac output divided by heart rate.

Sympathetic bursts in the integrated neurogram were identified by a custom-manufactured automated analysis program (Kienbaum et al. 2001); burst identification was then corrected by visual inspection by a single investigator. The program compensated for baroreflex latency, and associated each sympathetic burst with the appropriate cardiac cycle. Resting MSNA is expressed as bursts $/ 100$ heart beats $=$ burst incidence, and bursts $/$ minute $=$ burst frequency.

\section{Statistics}

Group average data are expressed as mean \pm SEM. The relationships among breathing rate, MSNA and cardiovascular variables were assessed using linear regression analysis and Pearson's correlation coefficient was used. $P<0.05$ was accepted as statistically significant. 


\section{Results}

\section{Group averaged data for respiratory, cardiovascular and neural variables}

Group data are given in Table 2. In summary, breathing rate was similar in men and women but compared with men, women had significantly lower systolic, diastolic and mean blood pressures, cardiac output and stroke volume. Heart rate was higher in women. MSNA was lower in women than in men but the difference did not reach statistical significance (burst incidence: $P=0.08)$.

\section{Relationship between breathing frequency and MSNA}

There was a significant positive correlation between the number of breaths and MSNA (burst incidence) in men $(r=0.58, P<0.01)$ but not in women $(r=0.12, P>0.05$; Fig. 1$)$.

When MSNA was expressed as burst frequency, the tendency was similar in men but the relationship did not reach statistical significance $(r=0.39, P>0.05)$. In women there was no correlation between these variables $(r=0.10, P>0.05)$.

\section{Relationship between breathing rate and cardiovascular variables}

The vasoconstriction evoked by MSNA is likely to influence total peripheral resistance. Consistent with this idea we found that when breathing rate was related to total peripheral resistance there was a significant positive correlation in men $(r=0.65, P<0.01)$; however, this was not evident in women (Fig. 2, upper panels). Cardiac output also displayed a close (inverse) relationship to breathing rate in men $(r=-0.80, P<0.01)$ but not in women (Fig. 2 , middle panels). Cardiac output is a function of heart rate and stroke volume. In men, heart rate was inversely correlated with breathing rate $(r=-0.56, P=0.01)$, whereas there was no relationship in women (Fig. 2, bottom panels). For stroke volume, the relationships were not significant, neither in men $(r=-0.36, P>0.05)$ nor in women $(r=0.23, P>0.05)$.

In contrast, the sex-related differences for the relationship between respiratory rate and blood pressure were opposite to those shown in Figure 2. Thus, breathing rate showed significant positive correlations in women for diastolic (Fig. 3, right) and mean $(r=0.52, P$ $<0.05)$, but not for systolic $(r=0.29, P>0.05)$ blood pressures. In men, there was no correlation for diastolic (Fig. 3, left), mean $(r=0.06)$ or systolic $(r=0.10)$ pressures.

As body size has an influence on MSNA and possibly respiration, we correlated body mass and body mass index with MSNA and breathing rate. We found that there was no relationship of body mass to MSNA expressed as burst incidence (women: $r=0.03$; men: $r$ $=0.05$ ), MSNA expressed as burst frequency (women: $r=-0.11$; men: $r=0.08$ ) or breathing rate (women: $r=0.27$; men: $r=-0.23 ; P>0.05$ ). We also found no correlation of body mass index with MSNA expressed as burst incidence (women: $r=-016$; men: $r=$ 0.34 ), MSNA expressed as burst frequency (women: $r=-0.22$; men: $r=0.19$ ) or breathing rate (women: $r=0.17$; men: $r=0.05 ; P>0.05$ ).

\section{Discussion}

The main findings of the present study are (1) respiratory rate shows a remarkable set of relationships to resting levels of sympathetic and cardiovascular variables in young healthy humans and (2) these relationships are fundamentally different between men and women. In men we confirm the positive correlation between respiratory rate and MSNA, previously reported by Narkiewicz et al. (2006), but we found no such relationship in women. In addition, in men, but not in women, respiratory rate correlated inversely with cardiac output and heart rate and positively with total peripheral resistance. 


\section{Coupling between respiration and cardiovascular data}

In individual humans, close dynamic relationships between respiratory, sympathetic and cardiovascular variables are well established. Transient blood pressure variations (including those induced by respiration) are regularly associated with baroreflex-induced inverse changes in MSNA (Sundlöf \& Wallin 1977). In addition, there is also a clear blood pressureindependent coupling to respiration per se, so that during normal breathing the occurrence of sympathetic bursts declines during inspiration and increases again during expiration (Eckberg et al. 1985, Seals et al. 1990). Presumably, this respiratory rhythmicity is of primary central origin (Seals et al. 1993, Eckberg 2003).

The present findings suggest, however, that respiration also is coupled to cardiovascular regulation in a previously unrecognized way: In recumbent, resting men an association between average breathing rate and level of MSNA is reflected in significant relationships between average breathing rate and several basic cardiovascular measures. In women, on the other hand, we found no association between breathing rate and level of MSNA and then, breathing rate was usually not related to the cardiovascular variables. Why there was a correlation between breathing rate and diastolic blood pressure in women but not in men is unclear.

Spontaneous breathing frequency at rest is known to vary within very wide limits and in old publications, the range is given as 6-36 breaths $\mathrm{min}^{-1}$ in adults (cited by Benchetrit 2000). In a given individual the characteristics of respiration are similar from day to day (Golla \& Antonovich 1929, Morrow \& Vosteen 1953) and Dejours et al. (1961) introduced the term 'la personnalité ventilatoire' to indicate the reproducibility of a person's respiratory frequency, tidal volume and airflow shape. To measure a subject's respiratory characteristics is methodologically challenging because any measuring device tends to alter the pattern of breathing. Respiratory frequency has been found to be particularly reproducible (Shea et al. 1987), which may be because it can be obtained without a disturbing measuring device. These findings have been confirmed and extended in several later studies (Shea \& Guz 1992, Benchetrit 2000). Thus, an individual's respiratory pattern is reproducible over 4-5 years (Benchetrit et al. 1989), the inter-individual differences are present also during deep (stage 4) sleep (Shea et al. 1990) and, in a study of identical twins, it was concluded that the inter-individual differences are likely to be of genetic origin (Shea et al. 1989). So far, however, the central nervous mechanisms determining 'personnalité ventilatoire' are unknown.

In a way similar to that for the respiratory pattern, the strength of resting sympathetic vasoconstrictor outflow to muscle shows intra-individual reproducibility over long periods of time but varies widely (10-fold or more) among individuals (Sundlöf \& Wallin 1977, Fagius \& Wallin 1993, Charkoudian et al. 2005). Furthermore, in pairs of homozygotic twins, both individuals have been found to have similar levels of MSNA (Wallin et al. 1993), suggesting that these inter-individual differences also have a genetic background. Thus, in several respects 'tonic' respiratory and sympathetic vasoconstrictor drives have similar intra- and inter-individual characteristics.

\section{Underlying mechanisms}

Neurophysiological studies in animals (Bainton et al. 1985, Boczek-Funke et al. 1992, Häbler \& Jänig 1995) provide evidence of a strong link during the respiratory cycle between the outputs from inspiratory and expiratory respiratory neurones in the medulla oblongata on the one hand, and variations of efferent sympathetic nerve traffic on the other hand. This has led to the proposal of regulatory models in which the activities of respiratory and cardiovascular neurones control (Bachoo \& Polosa 1987), or are coupled to (Barman \& 
Gebber 1976), cardiovascular neuronal activities. Alternatively, respiratory and cardiovascular neurones are governed by a common pool of 'cardiorespiratory' interneurones (Richter \& Spyer 1990) which may be located in the caudal ventrolateral medulla (Mandel \& Schreihofer 2006). Our present findings in men are compatible with the idea that this type of interaction extends not only to dynamic but also to 'tonic' cardiorespiratory function. In other words, there may be an overriding cardiorespiratory influence, which regulates tonic levels of activity in the two systems in proportion to each other. Along these lines there is recent evidence of a central nervous upregulation of the respiratory-sympathetic coupling in neonatal spontaneously hypertensive rats (Simms et al. 2009). In humans, Narkiewicz et al. (2006) found a significant coupling between breathing rate and MSNA in healthy males. In addition, in patients with cardiac failure a relatively strong relationship between breathing rate and MSNA has been reported (Naughton et al. 1998) but as no healthy control group was included, it is unclear whether this was a physiological finding or evidence of an upregulated respiratory-sympathetic coupling.

The reason for our finding of sex-related differences in respiratory sympathetic coupling is unclear. In a study of 25 men and 25 women (Shea et al. 1987), men had larger forced ventilatory capacity, tidal volume and minute ventilation than women, so one possibility would be that sex-related differences in afferent signalling lead to differences in cardiovascular regulation.

Another possibility would be direct central nervous influence of steroid sex hormones (including both oestrogen and progesterone) on the control of respiration. Several such effects have been reported (for a review, see Behan et al. 2003) and as an example, oestrogen receptors have recently been found on respiratory motor neurones in the medulla oblongata (Behan \& Thomas 2005).

The effects of oestrogen on peripheral blood vessels may also contribute to the absence of correlation to cardiovascular parameters in women. For example, oestrogen has a direct vasodilatory effect which might compete with sympathetic vasoconstriction (Gilligan et al. 1994, Volterrani et al. 1995). Furthermore, oestrogen is known to increase the bioavailability of the vasodilator nitric oxide (Sudhir et al. 1996) and beta-2-mediated vasodilatation is greater in women than in men (Kneale et al. 2000).

\section{Limitations}

We do not claim that respiratory rate is the primary factor linking respiratory and sympathetic outputs, other aspects of the respiratory pattern may also be important. However, the experiments reported in the present study were not designed to study primarily respiratory function and therefore breathing rate was the only recorded respiratory variable. Thus, additional studies are required to clarify the mechanisms underlying the striking findings.

It is also possible that the observed sex-related differences in the relationships of breathing rate to neural-haemodynamic variables might be due to differences in body size and not sex hormones per se. However, we found that body mass and body mass index was not related to breathing rate or MSNA in men and women. This suggests that body size was not a factor involved in the observed sex-related differences.

In summary, there are important differences between young men and women in relationships between breathing rate, sympathetic nerve activity and haemodynamic variables. The positive relationship between breathing rate and MSNA in young men, and the inverse relationship between breathing and cardiac output, suggest that influences of respiration may be important for 'tonic' neural-haemodynamic balance. The lack of relationships among 
these variables in women reinforces our recent conclusion that there are fundamental differences between the sexes in terms of basic blood pressure regulation (Hart et al. 2009).

\section{Acknowledgments}

This study was supported by NIH HL-083947, NIH HL-83947 and NIH CTSA RR-024150 and Swedish Medical Research Council Grant 12170. We thank Tomas Karlsson, Shelly Roberts, Karen Krucker, Shirley Kingsley Berg, Jessica Sawyer and Nicholas Strom for their assistance in the conduct of the studies and analysis of the data.

\section{References}

Bachoo M, Polosa C. Properties of the inspiration-related activity of sympathetic preganglionic neurones of the cervical trunk in the cat. J Physiol 1987;385:545-564. [PubMed: 3656168]

Bainton CR, Richter DW, Seller H, Ballantyne D, Klein JP. Respiratory modulation of sympathetic activity. J Auton Nerv Syst 1985;12:77-90. [PubMed: 3980922]

Barman SM, Gebber GL. Basis for synchronization of sympathetic and phrenic nerve discharges. Am J Physiol 1976;231:1601-1607. [PubMed: 998806]

Behan M, Thomas CF. Sex hormone receptors are expressed in identified respiratory motoneurones in male and female rats. Neuroscience 2005;130:725-734. [PubMed: 15590155]

Behan M, Zabka AG, Thomas CF, Mitchell GS. Sex steroid hormones and the neural control of breathing. Respir Physiol Neurobiol 2003;130:249-263. [PubMed: 12853015]

Benchetrit G. Breathing pattern in humans: diversity and individuality. Resp Physiol 2000;122:123129.

Benchetrit G, Shea SA, Pham Dinh T, Bodocco S, Baconnier P, Guz A. Individuality of breathing patterns in adults assessed over time. Resp Physiol 1989;75:199-210.

Boczek-Funke A, Dembowsky K, Häbler HJ, Jänig W, Michaelis M. Respiratory-related activity patterns in preganglionic neurons projecting into the cat cervical sympathetic trunk. J Physiol 1992;457:277-296. [PubMed: 1297836]

Charkoudian N, Joyner MJ, Johnson CP, Eisenach JH, Dietz NM, Wallin BG. Balance between cardiac output and sympathetic nerve activity in resting humans: role in arterial pressure regulation. J Physiol 2005;568:315-321. [PubMed: 16037092]

Charkoudian N, Joyner MJ, Sokolnicki LA, Johnson CP, Eisenach JH, Dietz NM, Curry TB, Wallin BG. Vascular adrenergic responsiveness is inversely related to tonic activity of sympathetic vasoconstrictor nerves in humans. J Physiol 2006;572:821-827. [PubMed: 16513672]

Dejours P, Bechtel-Labrousse Y, Monzein P, Raynaud J. Etude de la diversite' des re'gimes ventilatoires chez l'homme. J Physiol Paris 1961;53:320-321. [PubMed: 13721311]

Eckberg DL. The human respiratory gate. J Physiol 2003;584:339-352. [PubMed: 12626671]

Eckberg DL, Nerhed C, Wallin BG. Respiratory modulation of muscle sympathetic and vagal cardiac outflow in man. J Physiol 1985;365:181-196. [PubMed: 4032310]

Fagius J, Wallin BG. Long-term variability and reproducibility of resting human muscle nerve sympathetic activity at rest, as reassessed after decade. Clin Auton Res 1993;3:201-205. [PubMed: 8400820]

Gilligan DM, Badar DM, Panza JA, Quyyumi AA, Cannon RO III. Acute vascular effects of estrogen in postmenopausal women. Circulation 1994;90:786-791. [PubMed: 8044949]

Golla FL, Antonovich S. The respiratory rhythm in its relation to the mechanism of thought. Brain 1929;52:491-509.

Guyenet PG, Dernall RA, Riley TA. Rostral ventrolateral medulla and sympathorespiratory integration in rats. Am J Physiol 1990;259:R1063-R1074. [PubMed: 2173425]

Häbler HJ, Jänig W. Coordination of sympathetic and respiratory systems: neurophysiological experiments. Clin Exp Hypertens 1995;17:223-235. [PubMed: 7735271]

Hart EC, Charkoudian N, Wallin BG, Curry TB, Eisenach JH, Joyner MJ. Sex differences in sympathetic neural-hemodynamic balance: implications for human blood pressure regulation. Hypertension 2009;53:571-576. [PubMed: 19171792] 
Johnson BD, Beck KC, Proctor DN, Miller J, Dietz NM, Joyner MJ. Cardiac output during exercise by the open circuit acetylene washin method: comparison with direct Fick. J Appl Physiol 2000;88:1650-1658. [PubMed: 10797126]

Kienbaum P, Karlsson T, Sverrisdottir YB, Elam M, Wallin BG. Two sites for modulation of human sympathetic activity by arterial baroreceptors? J Physiol 2001;531:861-869. [PubMed: 11251065]

Kneale BJ, Chowienczyk PJ, Brett SE, Coltart DJ, Ritter JM. Gender differences in sensitivity to adrenergic agonists of forearm resistance vasculature. J Am Coll Cardiol 2000;36:1233-1238. [PubMed: 11028476]

Mandel DA, Schreihofer AM. Central respiratory modulation of barosensitive neurones in rat caudal ventrolateral medulla. J Physiol 2006;572:881-896. [PubMed: 16527859]

Minson CT, Halliwill JR, Young TM, Joyner MJ. Influence of the menstrual cycle on sympathetic activity, baroreflex sensitivity, and vascular transduction in young women. Circulation 2000;101:862-868. [PubMed: 10694525]

Morrow PE, Vosteen RE. Pneumotachographic studies in man and dog incorporating a portable wireless transducer. J Appl Physiol 1953;5:348-360. [PubMed: 13022602]

Narkiewicz K, Phillips BG, Kato M, Hering D, Bieniaszewski L, Somers VK. Gender selective interaction between aging, blood pressure, and sympathetic nerve activity. Hypertension 2005;45:522-525. [PubMed: 15767469]

Narkiewicz K, van de Borne P, Montano N, Hering D, Kara T, Somers VK. Sympathetic neural outflow and chemoreflex sensitivity are related to spontaneous breathing rate in normal men. Hypertension 2006;47:51-55. [PubMed: 16344363]

Naughton MT, Floras JS, Rahman MA, Jamal M, Bradley TD. Respiratory correlates of muscle sympathetic nerve activity in heart failure. Clin Sci 1998;95:277-285. [PubMed: 9730846]

Richter, DW.; Spyer, KM. Cardiorespiratory control. In: Loewy, AD.; Spyer, KM., editors. Central Regulation of Autonomic Function. Oxford University Press; New York: 1990. p. 189-207.

Seals DR, Suwarno NO, Dempsey JA. Influence of lung volume on sympathetic nerve discharge in normal humans. Circ Res 1990;67:130-141. [PubMed: 2364488]

Seals DR, Suwarno NO, Joyner MJ, Iber C, Copeland JG, Dempsey JA. Respiratory modulation of muscle sympathetic nerve activity in intact and lung denervated humans. Circ Res 1993;72:440 454. [PubMed: 8418993]

Shea SA, Guz A. Personnalité ventilatoire - an overview. Respir Physiol 1992;87:275-291. [PubMed: 1604053]

Shea SA, Walter J, Murphy K, Guz A. Evidence for individuality of breathing patterns in resting healthy man. Respir Physiol 1987;68:331-344. [PubMed: 3616179]

Shea SA, Benchetrit G, Pham Dinh T, Hamilton RD, Guz A. The breathing pattern of identical twins. Respir Physiol 1989;75:211-224. [PubMed: 2711052]

Shea SA, Horner RK, Benchetrit G, Guz A. The persistence of a respiratory 'personality' into stage IV sleep in man. Respir Physiol 1990;80:33-44. [PubMed: 2367750]

Simms AE, Paton JFR, Pickering AE, Allen AM. Amplified respiratory-sympathetic coupling in the spontaneously hypertensive rat: does it contribute to hypertension? J Physiol 2009;587:597-610. [PubMed: 19064613]

Sudhir K, Jennings GL, Funder JW, Komesaroff PA. Estrogen enhances basal nitric oxide release in the forearm vasculature in perimenopausal women. Hypertension 1996;28:330-334. [PubMed: 8794812]

Sundlöf G, Wallin BG. The variability of muscle nerve sympathetic activity in resting recumbent man. J Physiol 1977;272:383-397. [PubMed: 592196]

Sundlöf G, Wallin BG. Human muscle nerve sympathetic activity at rest. Relationship to blood pressure and age. J Physiol 1978;274:621-637. [PubMed: 625012]

Volterrani M, Rosano G, Coats A, Beale C, Collins P. Estrogen acutely increases peripheral blood flow in postmenopausal women. Am J Med 1995;99:119-122. [PubMed: 7625415]

Wallin BG, Kunimoto M, Sellgren J. Possible genetic influence on the strength of human muscle nerve sympathetic activity at rest. Hypertension 1993;22:282-284. [PubMed: 8349319] 


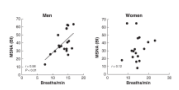

Figure 1.

Relationships between breathing rate and MSNA (BI = burst incidence $=$ number of bursts/ 100 heart beats) in men and women. 


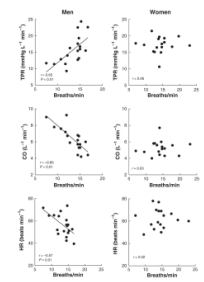

Figure 2.

Relationships between breathing rate and total peripheral resistance (TPR; upper diagrams), cardiac output (CO; middle diagrams) and heart rate (HR; bottom diagrams). 


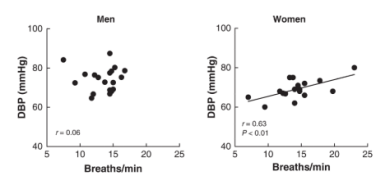

Figure 3.

Relationships between breathing rate and diastolic blood pressure (DBP). 


\section{Table 1}

General characteristics of the study groups

\begin{tabular}{lrc}
\hline & Men & Women \\
\hline Age & $27 \pm 1$ & $25 \pm 1$ \\
Height $(\mathrm{cm})$ & $180 \pm 3$ & $170 \pm 2^{*}$ \\
Weight $(\mathrm{kg})$ & $79 \pm 3$ & $65 \pm 2^{*}$ \\
BMI & $25 \pm 1$ & $22 \pm 1^{*}$ \\
\hline
\end{tabular}

BMI, body mass index.

${ }^{*} P<0.05$. 
Table 2

Cardiovascular and neural variables in men $(n=19)$ and women $(n=17)$

\begin{tabular}{|c|c|c|}
\hline & Men & Women \\
\hline HR (beats $\min ^{-1}$ ) & $56 \pm 2$ & $62 \pm 2^{*}$ \\
\hline $\mathrm{SBP}(\mathrm{mmHg})$ & $139 \pm 2$ & $127 \pm 1^{*}$ \\
\hline $\mathrm{DBP}(\mathrm{mmHg})$ & $74 \pm 1$ & $69 \pm 1^{*}$ \\
\hline MAP (mmHg) & $96 \pm 2$ & $89 \pm 1^{*}$ \\
\hline $\mathrm{SV}(\mathrm{mL})$ & $113 \pm 6$ & $85 \pm 4^{*}$ \\
\hline $\mathrm{CO}\left(\mathrm{L} \mathrm{min}^{-1}\right)$ & $6.3 \pm 0.3$ & $5.3 \pm 0.2^{*}$ \\
\hline TPR (mmHg L $\left.\min ^{-1}\right)$ & $15.7 \pm 1.0$ & $17.1 \pm 0.6$ \\
\hline MSNA (bursts $\min ^{-1}$ ) & $22 \pm 2$ & $19 \pm 2$ \\
\hline MSNA (bursts $100 \mathrm{hb}^{-1}$ ) & $40 \pm 3$ & $33 \pm 4$ \\
\hline Breathing rate (breaths $\min ^{-1}$ ) & $13.5 \pm 0.5$ & $14.0 \pm 0.9$ \\
\hline
\end{tabular}

Values are mean \pm SEM. HR, heart rate; SBP, systolic blood pressure; DBP, diastolic blood pressure; MAP, mean arterial pressure; SV, stroke volume; $\mathrm{CO}$, cardiac output; TPR, total peripheral resistance; MSNA, muscle sympathetic nerve activity.

Different from men $(P<0.05)$. 